\title{
Secondary Rhinoplasty on Patients with Cleft Lip and Palate: A Review
}

\author{
Vijayaranghan Sathyabama ${ }^{1}$, Muthusubramanian Veerabahu ${ }^{2}$
}

\begin{abstract}
Aim: The cleft lip and palate patients undergo a series of surgical procedures from the time of their birth, wherein the primary cleft lip correction procedure is carried out. The last in the series is rhinoplasty after the maxillary skeletal base has been set right in its dynamics with the mandibular base. The degree of deformity lies in the type of cleft and its accompanying features. The timing of correction at the primary lip closure stage, allowing a repositioning of the lateral nares, the detailed examination of the nose at the time of presentation ascertains the degree of augmentation necessary.

Background: The first mention of rhinoplasty dates back to the dates of Shusruta and his disciples who managed to reconstruct parts of the nose and ear, which were sliced as a part of criminal punishment. The Roman encyclopedist published the techniques of reconstruction, followed by the Europeans. By the 11th century, the Arabs converted Shushruta's book into Sanskrit, and this spread to the Western atmosphere. Evidence for a free flap graft and a forehead graft that were adopted and published by Doctors Cruzo and Findlay in 1794 are the first steps to the modern rhinoplasty techniques.

Review results: The correction of the nose at the primary surgery of the cleft lip was widely accepted to reduce the gross deformities that would manifest if the small corrections were not made appropriately the first time. The caudal resection of septae was identified as the reason for the growth deformities that happened secondarily. The secondary rhinoplasty was to be performed as a final procedure after the growth phase when all other surgical procedures were done and dealt with leaving an L-shaped strut of the septae at the dorsal, and the caudal end was advocated for a stable result of the septum without any buckling and further deformity.

Conclusion: The key to performing a near-perfect procedure lies in the clinical assessment at the time of presurgical presentation.

Keywords: Augmentation grafts, Cleft lip and palate, Columella lengthening, Rhinoplasty, Secondary rhinoplasty, Steal technique, Strut graft. International Journal of Head and Neck Surgery (2020): 10.5005/jp-journals-10001-1386
\end{abstract}

\section{INTRODUCTION}

Rhinoplasty is quite a challenging procedure to the oral and maxillofacial surgeons, and a cleft rhino is even further challenging, as it requires an immense understanding of the cleft as well as the esthetic concerns of the rhinoplasty.

During the developmental process, the median nasal process, with its globular process, forms the septal cartilage, the philtrum, and the premaxilla. ${ }^{1}$ Defective fusion of the median nasal process with that of the lateral process culminates in a cleft lip either unilaterally or bilaterally. ${ }^{2}$ On the contrary, the palate is formed by the fusion of the two palatine shelves at the midline and with that of the premaxilla anteriorly.

Etiology of cleft lip and palate has been attributed to both genetic and environmental factors. Deficiency of folic acid, maternal smoking during pregnancy, alcohol, and retinoic acid are some of the factors that contribute to the etiology of the cleft (Fig. 1).

The degree of nasal deformity visible after the cleft lip correction and the associated functional problems are a challenge to have them corrected at the primary surgery performed. The characteristic features of a unilateral cleft lip and palate are enlisted in Table $1 .^{1,3-5}$ The correction of a bilateral cleft is complex, and its features to be addressed are enlisted in Table 2. The existing cosmetic defect and the impaired function dictate the treatment planning at the later stage of treatment (Fig. 2).

The timing of the procedure was a controversy until the last decade was a consensus had been drawn by the facial plastics ${ }^{6}$ to go ahead with the primary correction of the rhinoplasty soon after the primary lip correction. ${ }^{7,8}$ The follow-up surgery that would
1,2Department of Oral and Maxillofacial Surgery, Ragas Dental College
and Hospital, Chennai, Tamil Nadu, India

Corresponding Author: Vijayaranghan Sathyabama, Department of Oral and Maxillofacial Surgery, Ragas Dental College and Hospital, Chennai, Tamil Nadu, India, Phone: +91 9840297029, e-mail: satvarnan@gmail.com

How to cite this article: Sathyabama V, Veerabahu M. Secondary Rhinoplasty on Patients with Cleft Lip and Palate: A Review. Int J Head Neck Surg 2020;11(2):26-31.

Source of support: Nil

Conflict of interest: None

happen at the end of all the series of surgeries by the age of 21 was to correct any residual defect that occurs after the primary surgery and the defective growth that has happened since then (Fig. 3).

\section{History}

The cleft deformity dates back to the pre-Christian era of 390 BC. Graefe in 1816 and Roux in 1819 have published in detail, the corrective surgical procedure for cleft lip and palate. Jehen Yperman first described the unilateral and bilateral cleft and mentioned about the needle used to stabilize the cleft lip for the suturing. Germanicus Mirault first introduced the triangular flap to transposition the lateral aspect of the defect into the medial, thus augmenting the vertical reduction, which was later modified by CW Tennison in 1952 and Peter Randal in 1959. Werner Hagerdon 


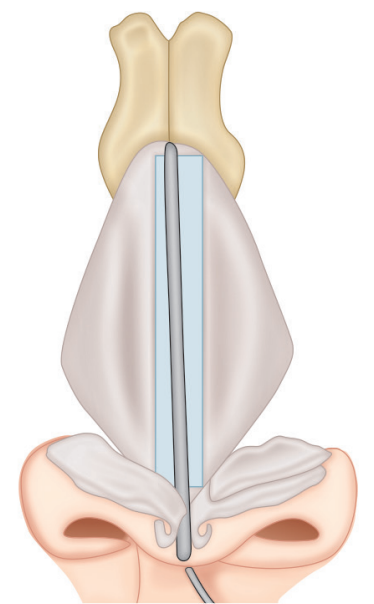

Fig. 1: Frontal view of nasal architecture

Table 1: Commonly associated features of unilateral cleft nose deformity

- Nose is elongated on the cleft side

- The lateral alar is defective and positioned inferiorly and laterally

- The dome is retro displaced

- Base of columella deviated away from the cleft

- Internal web of the nostril is more pronounced and reduces the nostril space on the cleft side anterior nasal spine is antero laterally displaced

- Medial crus of the cleft is short buckled away from the cleft reducing the nasal tip height

- Angle formed between the lateral and the medial crura is increased

Table 2: Commonly associated features of bilateral cleft nose deformity

- Nose is wide and the lateral cartilage are extended laterally and inferiorly

- Nasal tip is deflected inferiorly

- Overhanging columella that is depressed and short

- Premaxilla is separated and shifted anteriorly with the cleft running bilaterally on the hypoplastic maxilla

- Caudal septae are displaced inferiorly and posteriorly further improvised the triangular flap to a quadrangular one. ${ }^{9}$ Followed thereafter by Ralph Millard and then the other newer concepts followed (Fig. 4).

Cleft rhinoplasty, on the contrary, goes hand in hand in correcting the nasal deficit, Humby in 1938 described flipping the excess length of the lower lateral cartilage on the noncleft side to the deflected cleft side increasing the height of the medial crus uniformly (Fig. 5). ${ }^{10}$ Tajima also described suturing the lower lateral cartilage to the upper lateral cartilage to allow the correction of the deflection. ${ }^{10}$ Intra domal and interdomal sutures were given to reinstating the vertical height of the medial crura and the columellar length. Salyer and Kirschbaum in 1992, emphasized the plication and early repositioning of the lower lateral cartilage to prevent internal lateral webbing. McComb had done a 10-year review on his patients in whom he had repositioned the lateral cartilage at the primary repair and found that the results were satisfactory and were not hindering the secondary procedure (Fig. 6). ${ }^{11}$

\section{Anatomy with Manifestation of Cleft Lip and Nasal Deformity Unilateral Cleft Lip}

The unilateral cleft lip presents with the defective maxilla, an underdeveloped nasal piriform aperture with a deficient alar base. The nasal alar is shifted laterally and inferiorly. The medial crus is shortened, and the lateral crus of the lower lateral cartilage is extended laterally. The supra tip is ill-defined, and the columella shifted to the noncleft side. The former shifts the nasal septum to the noncleft side and the latter takes the alar base positioned inferior and lateral than the non-cleft side. ${ }^{12}$ This with the inherent deficiency of the basal piriform rim and the defective insertion of the orbicularis oris accentuates, the nasal deformity on the cleft side and reduces the nasal sill (Fig. 7).

\section{Bilateral Cleft Lip}

A bilateral cleft, on the contrary, has a symmetrical defect. The deficient soft tissue component bilaterally along with a complete absence of the bony piriform base with a septal and anterior nasal spine deviation that has taken place inferiorly results in an under projected tip with a broad nasal bridge and protruding premaxilla (Fig. 8). ${ }^{13}$

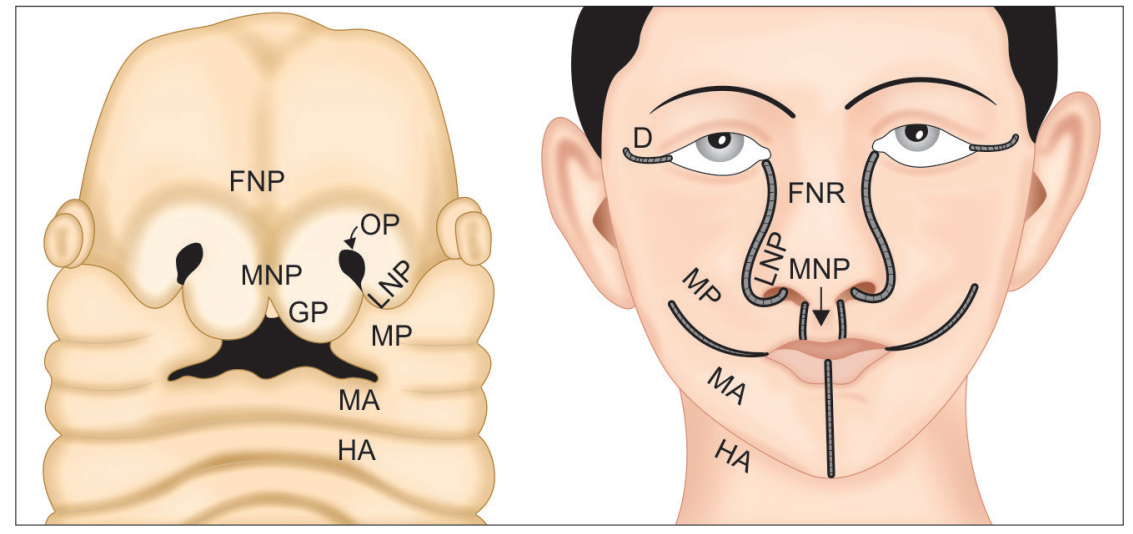

Fig. 2: Embryological development of the face. FNP, frontonasal process; MNP, median nasal process; MA, mandibular arch; HA, hyoid arch 


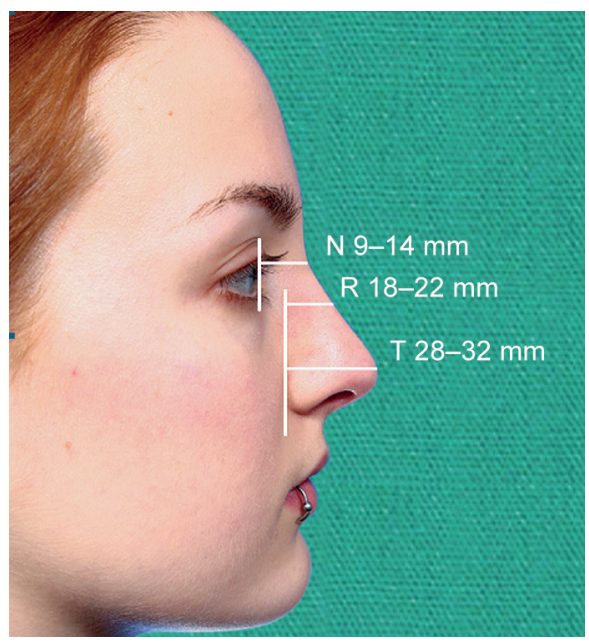

Fig. 3: Clinical assessment of a deviated nasal septum

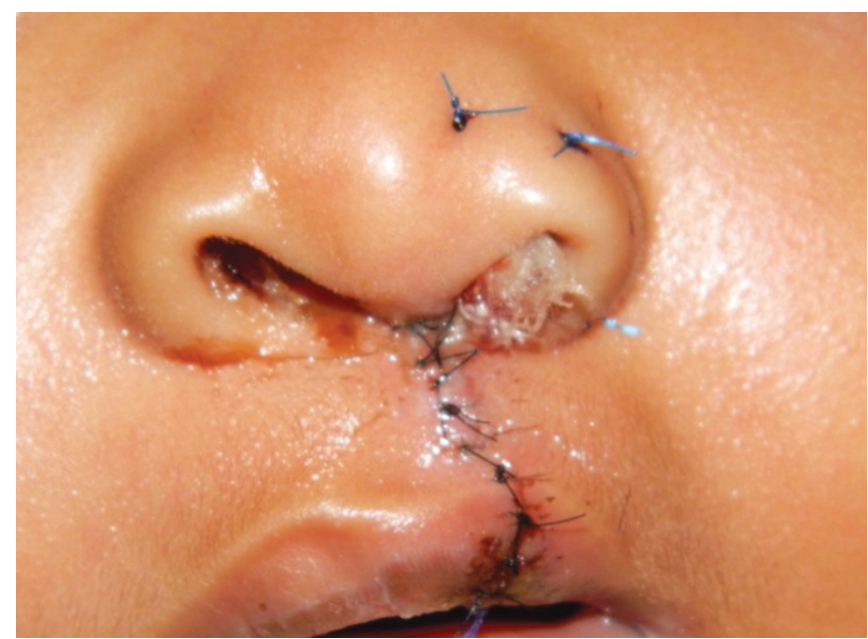

Fig. 5: Immediate postoperative view

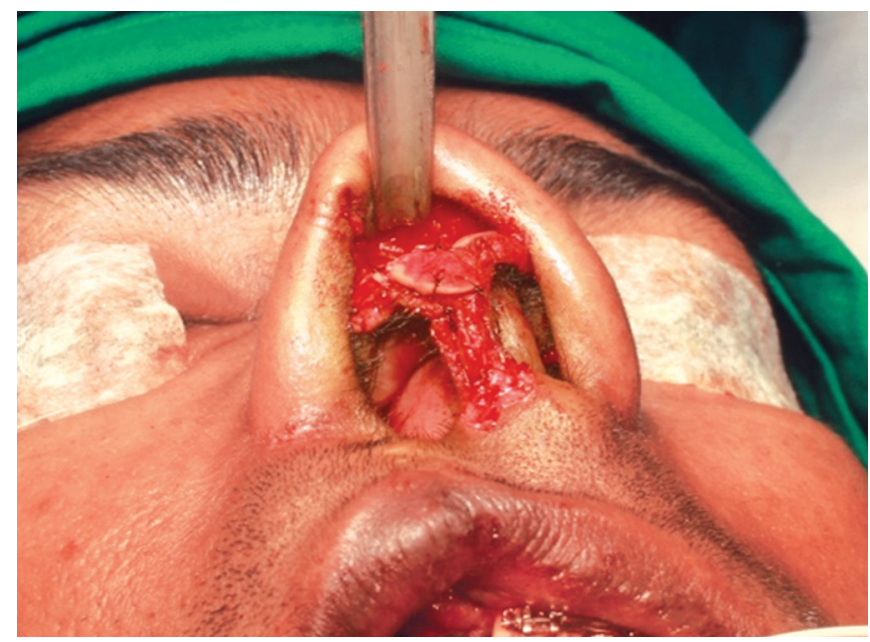

Fig. 7: Shield graft with interdomal and intradomal suturing with a columella strut graft to increase the columellar length

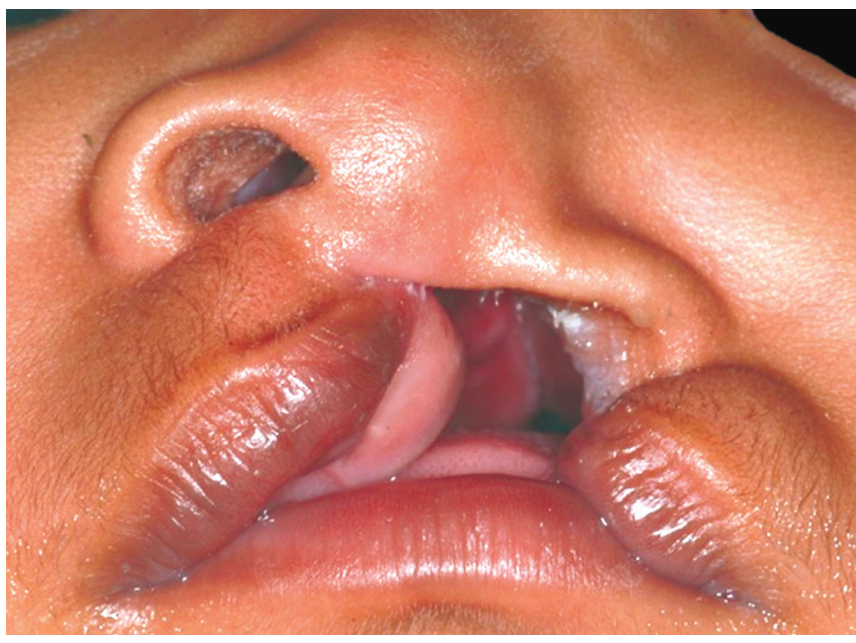

Fig. 4: Complete unilateral cleft lip and palate

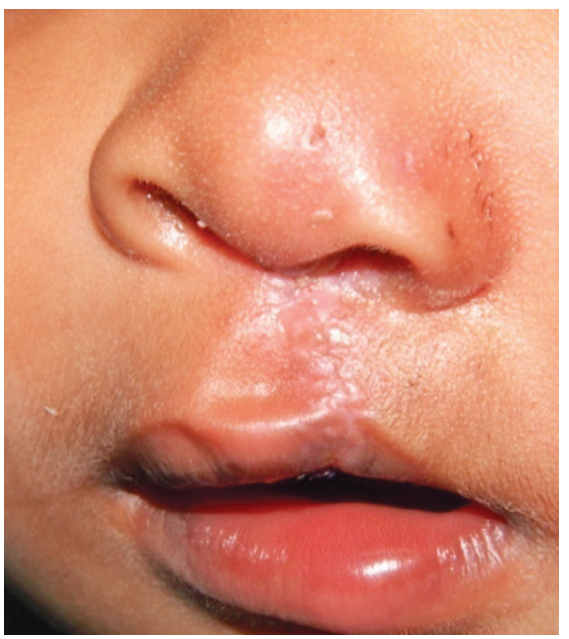

Fig. 6: Ten days old healing wound. Notice the approximation of the lateral crura

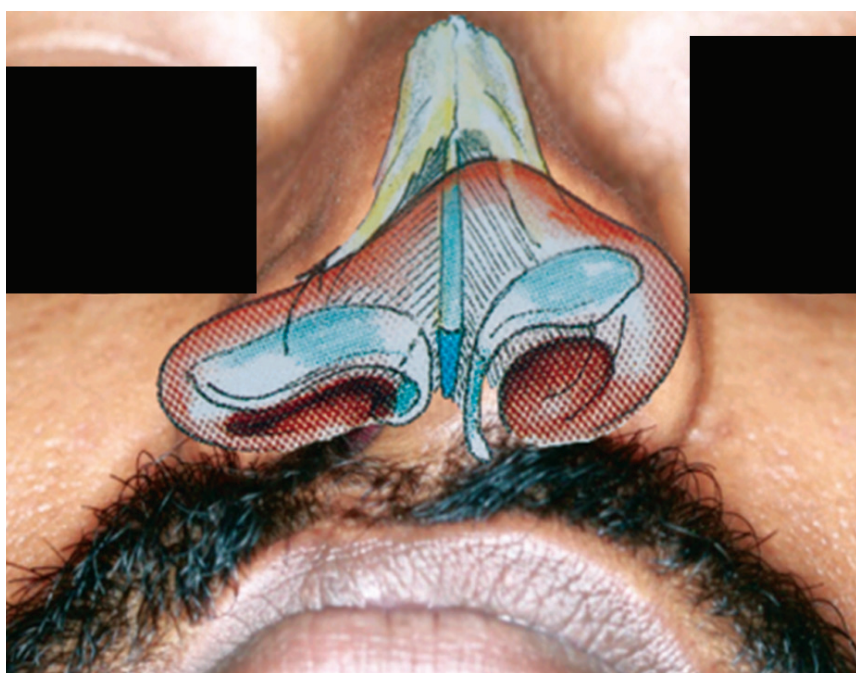

Fig. 8: Preoperative assessment for a secondary rhinoplasty 


\section{Timing of Rhinoplasty}

Several controversies exist over the timing of the primary rhinoplasty. Now it is accepted that the primary rhinoplasty be addressed along with the primary cleft lip, ${ }^{6,10,11,14-17}$ which allows the esthetics to be improved and then the residual defects addressed as a secondary procedure. ${ }^{4,5}$ The effects of the trimming of the caudal septae have been recognized, and the growth retardation has been addressed. The intermediate rhinoplasty is performed between 4 and 6 years of age. The basic defects are corrected without any augmentation. ${ }^{6}$ The secondary rhinoplasty happens at 14-16years of age with cartilage harvest if necessary (Fig. 9).

\section{Examination and Clinical Findings}

History and examination are of paramount importance to assess the patients' expectation and psychology that match the physical defects present with the patient. Most cleft patients are in need of esthetic and functional correction rhinoplasty. The dorsal height measures the height of the dorsum from three distinctive regionsthe nasion, the rhinion, the pronase (Fig. 10). ${ }^{18}$

The base should be in the shape of an equilateral triangle. ${ }^{19}$ The columella footplate complex to the lobule should be 2:1. ${ }^{18,20}$ The columella should curve down slightly from the nasal tip to the base.

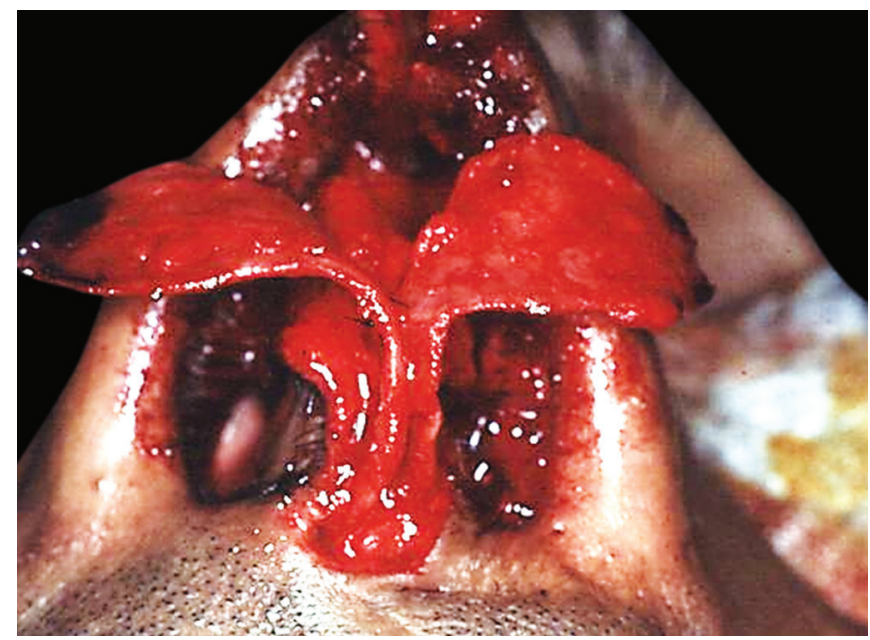

Fig. 9: Open rhinoplasty with a freed up lower lateral cartilage

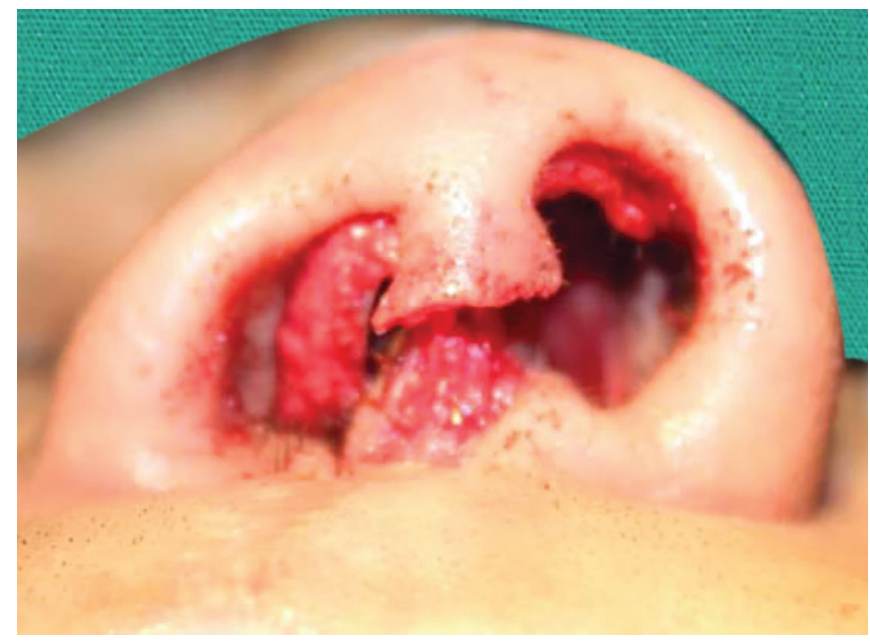

Fig. 11: Transcolumellar incision for open rhinoplasty prior to suturing
The internal nasal valve should have a minimum of $10^{\circ}$ to $15^{\circ}$ angulation with the weak nasal tip well supported. ${ }^{24}$ The vestibular webbing, if present, should be removed, and the alar base should be sufficiently supported (Fig. 11). ${ }^{10}$

\section{Anatomical Correction}

- Nasal root with the septum

- Nasal tip and the columella

- Lateral alar cartilage with the nasal sill correction

- Internal nasal valve and vestibular webbing.

\section{Nasal Root with the Septum}

The nasal root is wide with a deviation away from the cleft side. The correction of the nasal complex is performed after the maxillary hypoplasia has been corrected, and the skeletal bases have been repositioned at the desired levels with the completion of the orthodontic treatment (Fig. 12). ${ }^{5}$

The buckling of the septum towards the cleft side and the attachment of the septum at the anterior nasal spine away from the cleft side are corrected. An L-shaped strut with a minimum of $1.5 \mathrm{~cm}$ in the dorsal and the caudal portion was left behind to prevent the collapse of the septum by Killian. ${ }^{21,22} \mathrm{~A}$ swinging door correction of the septum could be done to the deviated attachment of the

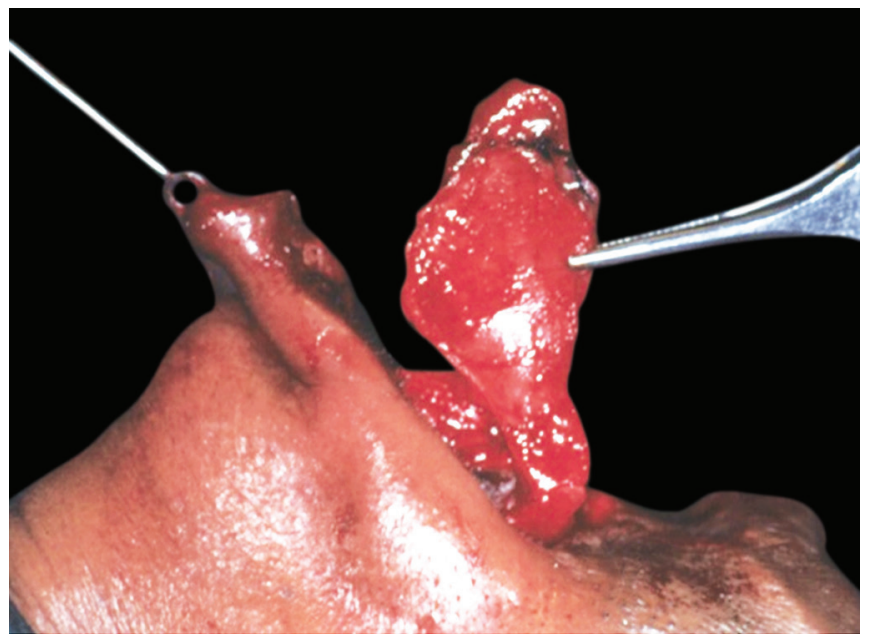

Fig. 10: Interdomal sutures to increase columella height

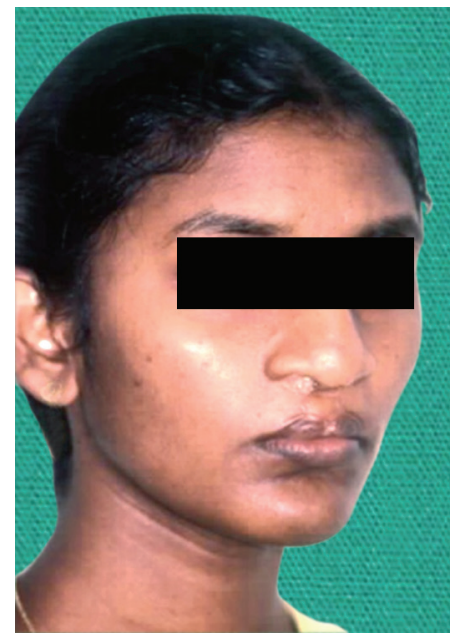

Fig. 12: Near-normal rhinoplasty 
septum to the anterior nasal spine, as described by Metzenbaum. Whatever be the technique that is employed to correct the deviated septum, the final suturing is done with polydioxanone anchoring the caudal septum to the nasal spine. ${ }^{22}$

Further augmentation of the septum is carried out using spreader grafts ${ }^{22,23}$ to improve the internal valve between the upper lateral cartilage and the septum. The concavity of the septum corrected with multiple scorings on the concave side with wedge resections on the convex side releasing any inbuilt memory that could be present with sutures placed both above and below the deviation.

The nasal dorsum that is flat and deviated away from the cleft side augmented with rib grafts on the dorsal aspect. The septal deviation corrected and supported with strut grafts that eliminate the deviation.

\section{Nasal Tip and the Columella}

The tip in a cleft patient is blunted, with the dome depressed and the columella shortened. The lateral crura of the lower lateral cartilage are extended flat and positioned inferiorly and caudally. Depending on the primary technique involved with the correction of the nose, the anatomy might present itself with different tip variations.

The nasal tip correction can be done with columellar strut graft, which increases the length of the columella, ${ }^{24-26}$ avoiding its width and increasing the tip projection. The medial crura are sutured with the septum intervening that lengthens the shortened medial crura on the cleft side. Lateral crural steal is performed to bring about symmetry in the height of the medial crura. ${ }^{6}$

Onlay batten grafts on the side of the cleft are to augment the displaced and the deficient lateral cartilage. ${ }^{27}$

In case of bilateral clefts, the flattened dome with its wideangle is reduced to give a good projected tip with a good nasal skeletal support framework. Spreader grafts ${ }^{24,27}$ are used if there is narrowing of the internal nasal valve. Finally, the tip height and the projection improved with a shield graft. ${ }^{6}$

\section{Lateral Alar Cartilage with the Nasal Sill Correction}

Nasal sill is often deficient with the cleft deformity and necessitates a bony graft to fill in the defect at the piriform aperture. ${ }^{6}$ With the base being supported and the maxilla repositioned, the rhinoplasty gets a better basal view and a profile view. The curved batten ${ }^{1}$ graft for the $a a^{6}$ and the common strut improve the esthetics.

The lateral alar flaring is then corrected with the repositioning of the alar by the Weir technique. This enhances the symmetry of the external nasal valve.

\section{Internal Nasal Valve and Vestibular Webbing}

If these procedures reduce the internal nasal valve then spreader grafts are given to increase the volume of the internal nasal valve. Micronostril should be corrected using a perialar nasolabial flap and should be avoided at the primary surgery.

The lateral webbing are removed by releasing the nasomaxillary ligaments and repositioning the alar base at the primary surgery. 22,28

\section{SUMMARY}

Secondary rhinoplasty is a very challenging procedure to restore esthetics form and function of the nasal architecture. The hard, bony, and cartilaginous defects should be corrected. The nasal root, dorsum, and the nasal tip to be synchronous to the corrected skeletal base. The septum, upper lateral, and lower cartilages should be in alignment. The soft tissues corresponding to the philtrum, columella, and the alar base to be symmetrical to each other. The external nasal valve and the internal nasal valve to be pronounced. The primary correction that is done should usually augment the secondary procedure.

\section{ACKnOWLedgments}

The surgical team members at Ragas Dental College and Hospital for their constant support and encouragement.

\section{References}

1. Gary C, Sykes J. Intermediate and definitive Cleft Rhinoplasty. Facial Plast Surg Clin North Am 2016;24:487-494. DOI: 10.1016/ j.fsc.2016.06.017.

2. Millard JR. Cleft Craft: Bilateral and Late Deformities, 1st ed., Boston, Mass: Little, Brown; 1976.

3. McComb HK, Coghlan B. Primary repair of the Unilateral Cleft lip and Nose, Completion of a Longitudinal Study. Cleft Palate Craniofac J 1996 Jan;33(1):23-30. DOI: 10.1597/1545-1569_1996_033_0023_ protuc_2.3.co_2.

4. Jonnalagadda A, Jami S, Pydi KL. Cleft Lip Rhinoplasty. Sci J Clin Med 2016;5(4-1):20-26.

5. Cohen M, Morris DE, White AD, et al. Functional and aesthetic correction of secondary unilateral cleft lip nasal deformities. Indian J Plast Surg 2009 Oct;42(Suppl):S91-S101. DOI: 10.4103/09700358.57195 .

6. Cuzalina A, Jung C. Rhinoplasty for the Cleft Lip \& Palate patient. Oral Maxillofac Surg Clin North Am 2016 May;28(2):189-202. DOI: 10.1016/ j.coms.2015.12.002.

7. Haddock NT, McRae MH, Cutting CB. Long term effect of primary cleft rhinoplasty on secondary cleft rhinoplasty in patients with unilateral cleft lip - cleft palate. Plast Reconstr Surg 2012;129(3):740-748. DOI: 10.1097/PRS.0b013e3182402e8e.

8. Sykes JM, Jang YJ. Cleft Lip Rhinoplasty. Facial Plast Surg Clin North Am 2009 Feb;17(1):133-144. DOI: 10.1016/j.fsc.2008.10.002.

9. Bhattacharya S, Khanna V, Kohli R. Cleft Lip:The historical perspective. Indian J Plast Surg 2009 Oct;42(Suppl):S4-S8. DOI: 10.4103/09700358.57180 .

10. Van Beek AL, Hatfield AS. Ellie Shpnef: Cleft Rhinoplasty. American Society of Plastic Surgeons; 2004.

11. McComb H. Primary correction of Cleft lip and nasal deformity. A 10 year review. Plast Reconstr Surg 1985 June;75(6):791-799. DOI: 10.1097/00006534-198506000-00003.

12. Blair VP. Nasal deformities associated with congenital cleft of the lip. JAMA 1925;84:185-187.

13. Hellings PW, Hens G, Shoenaers J, et al. Unique approach to secondary cleft lip rhinoplasty in facial plastic surgery. B-ENT 2010;6(Suppl 15):97-101.

14. Salyer KE. Early and late treatment of Unilateral cleft nasal deformity. Cleft Palate Craniofac J 1992 Nov;29(6):556-569. DOI: 10.1597/15451569_1992_029_0556_ealtou_2.3.co_2.

15. Trott JA, Mohan N. A preliminary report on open tip rhinoplasty at the time of lip repair in unilateral cleft lip and palate: The Alor Setar experience. Br J Plast Surg 1993 Jul;46(5):363-370. DOI: 10.1016/00071226(93)90040-i.

16. Mulliken JB. Primary repair of bilateral cleft lip and nasal deformity. Plast Reconstr Surg 2001 Jul;108(1):181-194. DOI: 10.1097/00006534200107000-00028.

17. Capone R, Sykes J. Evaluation and management of Cleft Lip and Palate disorders. Facial Plastics and Reconstructive surgery. NY: Thieme; 2009. pp. 1059-1060.

18. Khan HA. Rhinoplasty: Initial Consultation and Examination. Oral Maxillofac Surg Clin North Am 2012 Feb;24(1):11-24. DOI: 10.1016/ j.coms.2011.11.002. 
19. Bohluli B, Moharamnejad N, Yamini A. Nasal base surgery. Oral Maxillofac Surg Clin North Am 2012 Feb;24(1):87-94. DOI: 10.1016/ j.coms.2011.10.009.

20. Ghavami A, Janis JE, Acikel C, et al. Tip shaping in Primary Rhinoplasty: An algorithmic approach. Plast Reconstr Surg 2008 Oct;122(4): 1229-1241. DOI: 10.1097/PRS.0b013e31817d5f7d.

21. Parrilla C, Artuso A, Gallus R, et al. The Role of Septal surgery in cosmetic Rhinoplasty. Acta Otorhinolaryngol Ital 2013 Jun;33(3): $146-153$.

22. Nease CJ, Deal RC. Septoplasty in conjunction with Cosmetic Rhinoplasty. Oral Maxillofac Surg Clin North Am 2012 Feb;24(1):49-58. DOI: 10.1016/j.coms.2011.10.006.

23. Kaufman $Y$, Buchanan EP, Wolfsinkel, EM, et al. Cleft nasal deformity and Rhinoplasty. Semin Plast Surg 2012 Nov;26(4):184-190. DOI: 10.1055/s-0033-1333886.
24. Bagheri SC. Primary Cosmetic Rhinoplasty. Oral Maxillofac Surg Clin North Am 2012 Feb;24(1):39-48. DOI: 10.1016/j.coms.2011. 10.001 .

25. Ralph Jr DR. Columella lengthening by a forked flap. Plast Reconstr Surg Transplant Bull 1958 Nov;22(5):454-457.

26. Takato $T$, Yonehara $Y$, Susami T. Columella lengthening using a cartilage graft in the bilateral cleft lip-associated nose: choice of cartilage according to age. J Oral Maxillofac Surg 1995 Feb;53(2): 149-157. DOI: 10.1016/0278-2391(95)90393-3.

27. Koehler J, Mclain L. Grafting in Cosmetic Rhinoplasty. Oral Maxillofac Surg Clin North Am 2012 Feb;24(1):59-66. DOI: 10.1016/ j.coms.2011.10.010.

28. Narayanan V, Adenwalla HS. Primary Rhinoplasty at the time of unilateral cleft lip repair: A review and our protocol. Puthucode J Cleft Lip Palate Craniofac Anomal 2015;2:92-97. 